\title{
Le prédicat complexe faire + Vinf dans le langage enfantin entre 4 et 6 ans
}

\author{
Yanka Bezinska, Jean-Pierre Chevrot \& Iva Novakova \\ Laboratoire LIDILEM, Université Stendhal, Grenoble III \\ yanabez@yahoo.fr \\ ipchevrot@wanadoo.fr \\ inovakova@yahoo.fr
}

\section{Introduction}

Nombreuses sont les études menées sur l'emploi transitif (sens causatif) de verbes intransitifs chez les enfants (cf. Bowerman, 1974), ainsi que sur les constructions causatives et le processus de leur acquisition (Comrie, 1976; Shibatani, 1976; Ammon \& Slobin, 1979; Dixon, 2000). Toutefois, l'évolution du prédicat complexe faire + Vinf dans le langage enfantin a été peu étudiée, à notre connaissance (cf. les études de Labelle, 1984 ; Sarkar, 2002). La présente communication est consacrée à la construction factitive faire + Vinf chez des enfants monolingues francophones âgés de 4 à 6 ans. Elle se propose également de comparer les étapes de sa grammaticalisation à celles de son acquisition dans la recherche d'éventuelles correspondances, une question qui n'a pas fait l'objet d'un examen systématique. Notre étude s'inscrit à la fois dans le cadre de l'approche fonctionnelle pour l'analyse du prédicat complexe faire + Vinf et de l'approche cognitive (usage based approach, Tomasello, 2003) pour l'analyse des étapes d'acquisition de la construction.

Dans un premier temps, nous tenterons d'esquisser les étapes de grammaticalisation de la construction factitive faire + Vinf pour aboutir à son statut actuel de prédicat complexe. Nous présenterons par la suite l'expérimentation menée sur l'acquisition de faire + Vinf par des enfants monolingues francophones entre 4 et 6 ans et les premiers résultats obtenus.

\section{La grammaticalisation de faire + Vinf}

Depuis une date assez récente, les travaux sur le changement linguistique sont en plein essor (Hopper \& Traugott, 1993, Marchello-Nizia, 2001). Ce phénomène universel concernant tous les domaines de la langue est dû à un ensemble de facteurs : facteurs d'ordre cognitif, social ou pragmatique, facteurs internes au système de la langue (Combettes et al. 2003 : 234).

Toutefois, notre intérêt porte sur le changement d'ordre syntaxique ; il existe deux approches pour traiter ce phénomène : une approche formelle et une approche fonctionnelle (Peyraube, 2002: 47 - 49). La première considère que c'est l'acquisition par l'enfant de sa langue maternelle qui est à la base du changement grammatical, celui-ci étant non progressif et autonome de toute considération fonctionnelle. La seconde relie le changement syntaxique à des facteurs discursifs, historiques, développementaux. Peyraube (2002: 49 - 54) mentionne également les trois mécanismes sous-jacents au changement syntaxique observable dans les langues : analogie (extension), réanalyse (grammaticalisation) et emprunt externe. Parmi ces mécanismes, la grammaticalisation est le seul à pouvoir expliquer le processus de la formation du prédicat complexe (PC) faire + Vinf.

Le terme de grammaticalisation a été défini pour la première fois par A. Meillet (1912/1982: 131) comme « le passage de la composante lexicale à la composante grammaticale. ». Kurylowicz (1965: 52) élargit cette notion en parlant d' « un processus, qui soit change des lexèmes pleins en mots grammaticaux, soit rend des éléments grammaticaux encore plus grammaticaux. ». Dans la littérature, on retrouve également 
le terme de «grammaire émergente» (emergent grammar) désignant «un mouvement vers une structure » (movement toward a pattern) (Hopper, 1987 : 148).

Il existe quatre paramètres de nature différente (sémantique, morphosyntaxique, morphophonologique, phonétique), qui sont révélateurs du processus de grammaticalisation (Lamiroy, 1999 : 35 - 37). Pourtant, l'évolution de faire + Vinf n'illustre que deux de ces quatre paramètres : la désémantisation et la décatégorisation syntaxique de faire. Le premier processus concerne l'entrée lexicale qui «se vide progressivement de son sens plein pour acquérir en revanche un sens fonctionnel, grammatical» (Lamiroy, 1999 : 35). La décatégorisation syntaxique consiste dans le fait que « le verbe tend de moins en moins à sélectionner les syntagmes nominaux pour s'associer de plus en plus à des types de verbes non finis ou non tensés, tel l'infinitif. » (Heine, 1993 : 75). Finalement, une montée des clitiques se produit aboutissant à une réanalyse des deux verbes comme un seul syntagme verbal. Les travaux sur la grammaticalisation des unités linguistiques font état de trois phases, ce qui est également valable pour la construction faire $+\operatorname{Vinf}($ Chamberlain, 1986 ; Simone \& Cerbasi, 2001) :

Etape 1 : l'emploi généralisé en latin de constructions bi-clausales V1 (de commande, de persuasion) + complémenteur + V2 2 subjonctif (inducere aliquem ut mentiatur).

Etape 2 : les formes ancienne (deux prédicats) et nouvelle (prédicat complexe) coexistent en ancien et en moyen français $\left(\mathrm{XI}^{\mathrm{e}}\right.$ et $\mathrm{XV}^{\mathrm{e}}$ siècles $)$.

Etape 3: la nouvelle forme exclut l'ancienne.

La première étape illustre une période antérieure au changement observable en latin classique, une langue de basse orientation causative (poor causative-oriented language, Simone \& Cerbasi, $2001: 450$ ). Le premier verbe de la construction $V 1+u t+V 2$ subjonctif n'est pas spécifiquement causatif; il est teinté de différentes valeurs modales (coercition, persuasion, ordre). Pour relier les agents de deux actions différentes et pour exprimer les rapports spécifiques établis entre eux (hiérarchie, degré d'agentivité, degré de contrôle), le latin fait appel à des constructions moins grammaticalisées du type : iubere $+u t+$ $V$ subjonctif, facere $+u t+V$ subjonctif, inducere $+u t+V$ subjonctif $:$

(1) Tu etsi non potuisti ullo modo facere ut mihi illam epistulam non mitteres, tamen mallem non esse missam.

[Ah! Certes tu ne pouvais pas faire autrement que de me l'envoyer, mais j'aurais été certainement plus heureux à ce qu'elle ne m'eût pas été envoyée.] (Cicéron, Epistulae ad Atticum, T.2, livre XI, epis. 21, paragr. 1, p. 384).

L'évolution de ces constructions à deux verbes distincts se poursuit, marquée par la suppression du complémenteur $\boldsymbol{u} \boldsymbol{t}$ et l'apparition de l'infinitif à la place du subjonctif :

(2) Ut ille fidicinam fecit nescire esse emptam tibi.

[Comme il a laissé ignorer à la joueuse de lyre qu'on l'achetait pour toi.] (Plaute, Comédies (Epidicus), T. 3 , acte III, 30, p. 144).

Par ailleurs, on pourrait déjà relever en latin des exemples d'insertion d'un syntagme nominal (SN) entre le verbe faire et l'infinitif :

(3) Qui nati coram me cernere letum fecisti et patrios fœdasti funere uoltus.

[...qui m'as fait voir de mes yeux le trépas de mon enfant, qui as souillé de mort le visage d'un père !] (Virgile, Enéide, T. 1, livres I - IV, p. 59).

(4) Quæ tremulum faciunt membris existere motum.

[...qui provoquent le tremblement de tous ses membres.] (Lucrèce Carus, De rerum natura, T. 1, livres I - III, p. 97). 
En ancien français, le verbe causatif faire peut être suivi de l'infinitif d'un verbe transitif ou intransitif ${ }^{1}$ :

(5) Morz est mis niés, ki tant me fist cunquere.

[Il est mort, mon neveu, qui me conquit tant de terres.] (Roland, v. 2920, p. 287)2.

(6) En seintes flurs, il les facet gesir.

[...et que parmi les saintes fleurs il les fasse reposer.] (ibid, v. 1856, p. 207)3.

Cependant, en ancien et moyen français, l'insertion d'un syntagme nominal (SN) entre faire et l'infinitif est tout à fait ordinaire lorsque ce dernier n'est pas suivi d'un complément d'objet direct; on en trouve des exemples significatifs en vers, en prose, dans le théatre:

(7) Si l'orrat Carles, ferat l'ost returner.

[Charles l'entendra, il fera retourner l'armée.] (Roland, v. 1060, p.147).

(8) Ge ferai le baron venir... (Béroul, Tristan, v. 3085, XIII s. $)^{4}$.

(9) Et puis fisent toutes manières de gens, petits et grans, partir (Froissart, Chron., I, 313, t.IV, p. 63, I. $\left.29-30, \mathrm{XIV}^{\mathrm{e}} \mathrm{s}.\right)$.

(10) Pour faire tout le monde rire (théâtre du XV et du XVI ${ }^{\mathrm{e}} \mathrm{s}$.).

Le degré de cohésion des éléments de la construction faire + Vinf change au cours des siècles. Peu à peu le SN inséré entre faire et l'infinitif se déplace à droite de l'infinitif. Le verbe faire, de sens très général, se désémantise et se décatégorise progressivement. Il commence à admettre la montée du clitique, ce qui est un indice révélateur « d'une restructuration, c'est-à-dire d'une réanalyse des deux verbes comme un seul syntagme verbal, et donc d'une grammaticalisation » (Lamiroy, 1999 : 44).

La deuxième étape de la grammaticalisation de faire + Vinf se caractérise donc par la fluctuation entre l'ancienne forme (à deux prédicats) et la nouvelle (une seule unité prédicative, prédicat complexe) :

(11) M'annonçant que des dieux la juste bienveillance aura de mon costé fait tourner la balance (J. de Schelandre, Tyr et Sidon, ATF, T. 8, v. 28, p. 47, 1628) ${ }^{5}$.

(12) Qui [un serpent] siffle et fait grincer la dent envenimée (Desmarets de Saint-Sorlin, Clovis, 1657 1673, A. Haase, Ergaenzende Bemerkungen zur Synt. Des XVII. Jh's, Zs.f.fz.Spr., XI, p. 218).

(13) Qui facit les coquins mandier? (Rabelais, Pantagruel, III, 14. XVI ${ }^{\mathrm{e}} \mathrm{s}$ ).

(14) Ce sera vous qui de nos villes ferez la beauté refleurir, vous qui de nos haines civiles ferez la racine mourir (Malherbe, T. 1, v. 92 et 94, p. 49 ; Regnier, Lex., s. v ; faire, 3).

En dépit de ces fluctuations, la nouvelle forme de la construction factitive faire + Vinf s'impose. Finalement, la soudure syntaxique de faire et du verbe à l'infinitif s'établit comme norme depuis le XVII ${ }^{\mathrm{e}}$ siècle (Nilsson-Ehle, 1948). De nos jours, le statut de prédicat complexe (PC) attribué à cette construction fait l'objet d'un large consensus dans la littérature linguistique (Gaatone, 1976; Alsina, 1996 ; Abeillé \& Godard, 2003, Creissels, 2006) ${ }^{6}$. Par ailleurs, il est intéressant, que certains auteurs contemporains remettent le $\mathrm{SN}$ au milieu du PC, et ce pour des raisons stylistiques ${ }^{7}$ :

(15) Les mots de passion qui faisaient l'amant frôler son visage au papier satiné (A. Daudet, Sapho, $1884)^{8}$.

(16) Ce qui faisait Armand répondre à ceux qui lui demandaient... (A. Gide, Les faux Monnayeurs, 1925).

La grammaticalisation de la construction factitive faire $+\operatorname{Vinf}$ en français est un processus unidirectionnel allant des structures analytiques (à deux prédicats, en latin) aux formes synthétiques (prédicat complexe, en français contemporain); sur le plan sémantique, il y aurait également une tendance à la lexicalisation de la construction (ex. : faire voir $=$ montrer, faire mourir $=$ tuer, faire connaître $=$ annoncer $)$. 


\section{L'acquisition de faire + Vinf}

Comme indiqué dans l'introduction, la seconde section de notre communication est consacrée à l'expérimentation menée sur l'acquisition de faire + Vinf chez des enfants monolingues francophones entre 4 et 6 ans. Notre principale interrogation porte sur le fait de savoir dans quelle mesure les étapes de l'évolution de cette construction dans le langage enfantin pourraient reproduire celles de sa grammaticalisation.

\subsection{Méthodologie}

\subsubsection{Participants}

L'expérimentation menée sur l'acquisition du factitif français faire + Vinf inclut 40 enfants monolingues francophones au total ${ }^{9}$. Ceux-ci sont répartis en deux tranches d'âge : 20 enfants ( 9 filles +11 garçons) entre 4 et 5 ans $(\mathrm{T} 1=49-59$ mois $)$ et 20 enfants $(9$ filles +11 garçons $)$ entre 5 et 6 ans $(\mathrm{T} 2=60-73$ mois).

\subsubsection{Procédure de recueil des données}

Les enfants sont enregistrés avec MD enregistreur audio et filmés avec HDD caméscope une seule fois en milieu scolaire (à la maternelle). Il s'agit d'entretiens individuels d'une durée approximative de 30 minutes.

Le choix des verbes cibles de notre recherche n'a pas été fait de manière arbitraire. Il a été conditionné par deux facteurs principaux : 1/ les propriétés syntaxiques et sémantiques des verbes ; 2 / la fréquence avec laquelle ceux-ci apparaissent dans les constructions factitives (calculée dans le corpus FRANTEXT : 1245 textes sélectionnés pour la période de 1900 à 2007). Nous avons donc choisi des verbes de nature sémantique et syntaxique variée :

a) intransitifs - rire et pleurer (inergatifs agentifs) ${ }^{10}$; danser (inergatif de mouvement) ${ }^{11}$ et tomber (inaccusatif de changement de position) $^{12}$ (Hypothèse inaccusative de Perlmutter, 1978, Levin \& Rappaport, 1995). Dans ce cas-là, notre but est de voir si les enfants monolingues francophones vont supprimer le verbe factitif faire (ex. : saute ça, vas boire mon bébé, je danse le p'tit chat, cf. Sarkar, $2002: 191)$.

b) transitifs - boire et manger. Ceux-ci peuvent apparaître dans des structures intransitives (ex. : Le bébé boit / mange.) et transitives (ex. : Le bébé boit du lait; L'ours mange du miel). Dans le cas d'une dérivation causative, ces verbes entrent dans des constructions factitives plus ou moins complexes (ex. : manger $\rightarrow X$ fait manger $Y$, ou bien, $X$ fait manger $Y \grave{a} Z$ ). Notre expérimentation vise à explorer la capacité des jeunes locuteurs à produire les deux types de constructions respectivement avec deux ou trois actants : 1/ La maman fait boire / manger le bébé; 2/ La maman fait boire du lait au bébé. ; Le kangourou fait manger du miel au petit ours.).

c) causatifs lexicaux - tuer = faire mourir et montrer = faire voir. Pour ce qui est de ces deux verbes, notre objectif est d'explorer l'apparition des erreurs par surgénéralisation (ex.: Ulysse, il *fait montrer le village à Hercule.). Celles-ci marquent une étape importante dans le développement langagier des enfants. En faisant appel à leurs capacités cognitives générales, ainsi qu'à leurs habiletés socio-cognitives, les jeunes locuteurs se transforment en créateurs de nouvelles constructions (en syntaxe, ce phénomène se fait sentir à partir de 3 ans jusqu'à l'âge de 4 - 6 ans) (Tomasello, 2003).

Nous avons également veillé à ce qu'il y ait un verbe fréquent et un verbe moins fréquent au sein de chaque catégorie. Les deux facteurs complexité et fréquence jouent un rôle crucial dans le processus d'acquisition d'un fait de langue (Tomasello, 2003). Le tableau 1 ci-dessous illustre ces deux critères : 


\begin{tabular}{|c|c|c|c|c|}
\hline \multicolumn{3}{|c|}{ Classes de verbes à tester } & + fréquents & - fréquents \\
\hline \multirow[t]{4}{*}{ Intransitifs } & \multirow{2}{*}{$\begin{array}{l}\text { avec } \\
\text { faire }\end{array}$} & rire & 918 & \\
\hline & & pleurer & & 242 \\
\hline & \multirow{2}{*}{$\begin{array}{l}\text { sans } \\
\text { faire }\end{array}$} & tomber & 711 & \\
\hline & & danser & & 221 \\
\hline \multirow[t]{2}{*}{ Transitifs } & \multicolumn{2}{|c|}{ boire } & 243 & \\
\hline & \multicolumn{2}{|c|}{ manger } & & 123 \\
\hline \multirow{2}{*}{$\begin{array}{l}\text { Causatifs } \\
\text { lexicaux }\end{array}$} & \multicolumn{2}{|c|}{ tuer } & & 10488 \\
\hline & \multicolumn{2}{|c|}{ montrer } & 26273 & \\
\hline
\end{tabular}

Tableau 1 : Classes de verbes inclus dans le protocole expérimental selon leurs propriétés syntaxiques et leur fréquence

Pour mener l'expérimentation, nous avons conçu trois tâches : production, compréhension, imitation avec changement du lexique (McDaniel, McKee \& Smith Cairns, 1998).

La tâche de production est présentée sous forme de brefs clips vidéo (des extraits de dessins animés), que les enfants observent sur l'ordinateur. Ces clips montrent des situations potentiellement causatives où un personnage fait agir un autre. L'objectif de la tâche est de voir si, ou bien, à quel point les jeunes locuteurs entre 4 et 6 ans sont capables de produire correctement le PC faire + Vinf. Pour éliciter la construction factitive, nous posons trois questions principales toujours dans le même ordre: 1/ sur le causateur dans la situation causative (ex. : Qu'est-ce qu'elle fait, la grenouille? $\rightarrow$ Elle gonfle ses joues.) ; 2/ sur le causataire de la situation causative (ex. : Qu'est-ce qu'il fait, Tarzan ? $\rightarrow$ Il pleure.) ; 3/ sur ce que le causateur fait au causataire (ex. : Qu'est-ce que la grenouille fait à Tarzan ? $\rightarrow$ Elle le fait pleurer.). D'une part, cet ordre d'apparition des questions permet de sensibiliser l'enfant en attirant son attention, d'abord, sur les actions non causatives (questions 1 et 2), puis sur la situation causative (question 3). D'autre part, il nous permet de faire des hypothèses sur les étapes d'acquisition par l'enfant du PC faire + Vinf. En d'autres termes, si le factitif est produit spontanément, avant toutes nos questions, ou bien en réponse à la première question, alors il n'y aura pas de doute sur la disponibilité du PC faire + Vinf en production. Par contre, si le factitif apparaît suite à la troisième question, on pourrait avancer l'hypothèse que l'enfant a toujours besoin d'aide extérieure (d'indices) pour produire correctement cette construction complexe.

La tâche de compréhension se déroule sous forme de jeux d'interaction entre l'enquêtrice et l'enfant. Elle a pour objectif d'explorer la compréhension du prédicat complexe faire + Vinf chez les jeunes locuteurs. A cet effet, l'enquêtrice produit un énoncé (à la forme impérative ou interrogative) évoquant une situation causative et l'enfant est amené à jouer la scène à l'aide de figurines (papa, maman, bébé, grande sour, araignée, mouche, coccinelle, papillon) (ex. : Voilà un bébé et une maman. La maman fait boire le bébé. A toi maintenant, fais comme la maman!, ou bien, Est-ce que tu peux faire comme la maman?).

La tâche d'imitation avec changement du lexique se déroule également sous forme de jeux d'interaction entre l'enquêtrice et l'enfant. Son objectif est l'exploration de l'imitation productive chez les jeunes apprenants. L'enquêtrice réalise une action causative à l'aide de figurines (cf. supra) et, en même temps, elle produit le modèle de formation de la construction factitive. Puis elle répète la même action avec d'autres figurines. L'enfant est amené à reproduire le modèle adulte en impliquant de nouveaux items lexicaux. Cette tâche expérimentale pourrait être illustrée par le schéma suivant : $\underline{\mathbf{A}}$ factitif $\underline{\mathbf{B}} \rightarrow \underline{\mathbf{C}}$ même factitif $\underline{\mathbf{D}}$ (ex.: Regarde bien, je vais te montrer quelque chose: La maman fait boire le bébé. Et maintenant, dis-moi, qu'est-ce qu'il fait, le papa? (l'enquêtrice montre à l'enfant le papa qui fait boire la grande sour). $\rightarrow$ Le papa fait boire la grande sœur.).

$\mathrm{Au}$ cours de l'expérimentation, tous les verbes cibles sont conjugués à la $3^{\text {-ème }}$ pers. sing. prés. Pour éliminer le facteur de la fatigue, l'ordre de leur apparition dans les trois tâches expérimentales varie pour chaque sujet interviewé. Autrement dit, nous avons effectué des permutations circulaires pour chaque enfant à partir de l'ordre général à savoir : a/ rire, pleurer ; b/ tomber, danser ; c/ boire, manger ; d/ tuer, montrer. 


\subsubsection{Codage des réponses}

Pour évaluer les réponses des enfants en production et en imitation, nous avons établi une échelle comprenant six niveaux (de la valeur 5 à la valeur 0 ). La valeur 5 correspond à différents degrés de maitrise de la construction factitive ; dans ce cas-là, les réponses des enfants sont plus ou moins proches de la cible adulte attendue. La valeur 0 indique l'absence de réponse de la part de l'enfant, ou bien, une réponse, qui ne prend en compte aucun des éléments de la situation causative :

\section{niveau 5 - présence du verbe factitif faire}

5a - emploi canonique du prédicat complexe faire + Vinf avec arguments présents, justes et correctement placés (ex. : Le papa fait rire la fille; Il la fait rire.).

5b - emploi conventionnel de faire + Vinf, mais hésitation au niveau du genre des arguments (ex. : Le papa fait rire la fille. $\rightarrow$ *Elle la fait rire, Il *le fait rire, *Elle *le fait rire.).

$\mathbf{5 c}$ - faire + Vinf avec causataire incorrectement récupéré comme datif (ex. : Il *lui fait rire; Il fait manger *à l'ours ; Il *lui fait, *à la petite fille, manger), ou bien, avec causataire incorrectement récupéré comme objet (ex. : Elle *le fait boire son biberon; Il fait boire *la grande sœur de la tisane).

5d - faire + Vinf avec un argument juste et un/des argument/s manquant/s (ex. : Le papa fait rire $+\varnothing ; \varnothing$ + fait boire le biberon; $\varnothing+$ fait pleurer ; $\varnothing+$ le fait boire).

5e - insertion d'un SN entre faire et l'infinitif (ex. : Elle fait *le pleurer).

niveau 4 - présence du verbe lexical à l'infinitif ou conjugué

$4 \mathbf{a}$ - énoncés elliptiques où l'enfant ne produit que le verbe lexical à l'infinitif avec un seul argument (ex. : Pleurer le bébé).

$\mathbf{4 b}$ - ellipses réduites où l'enfant ne produit que le verbe lexical à l'infinitif; le verbe factitif faire et les deux arguments (causateur, causataire) ne sont pas exprimés (ex. : Tomber).

$4 \mathbf{c}$ - transitivation causative : le verbe lexical est conjugué et les deux arguments sont présents (ex. : Il *rit la fille ; Il *boit *à la sœur).

\section{niveau 3 - constructions moins grammaticalisées donner à manger /à boire}

3a - les arguments sont présents et justes (ex. : Le kangourou donne à manger (du miel) au petit ours.).

$\mathbf{3 b}$ - avec argument/s manquant/s (ex. : Il donne à manger $+\varnothing ; \varnothing+$ lui donne à boire) .

\section{niveau 2 - surgénéralisations}

2a - les arguments sont présents et justes (ex. : Il *fait casser le robot; Elle fait gonfler des bulles).

$\mathbf{2 b}-$ avec argument/s manquant/s (ex. : Il fait casser $+\varnothing ; \varnothing+$ fait casser le robot).

niveau 1 - réponses partielles; il s'agit de réponses portant uniquement sur l'action du causateur qui fait agir le causataire (ex. : La grande sour fait une grimace; La grenouille gonfle ses joues; Elle lui fait peur ; Elle donne le biberon au bébé), ou bien, sur celle du causataire (ex. : Le bébé rit ; Tarzan pleure).

niveau 0 - absence de réponse cohérente (ex. : Il a attaqué le robot, pour faire tomber; Le loup fait une chanson à la sorcière, pour faire danser).

En ce qui concerne le codage des causatifs lexicaux tuer et montrer, l'échelle que nous avons établie comprend cinq niveaux (de la valeur 4 à la valeur 0 ). La valeur 4 correspond à l'emploi conventionnel de ces deux verbes. La valeur 0 indique l'absence de réponse de la part de l'enfant, ou bien, une réponse, qui ne prend en compte aucun des éléments de la situation causative : 
niveau 4 - l'emploi des causatifs lexicaux

4a - avec arguments présents et justes (ex. : Hercule tue le lion ; Ulysse montre le village à Hercule ; Il montre avec son doigt).

$\mathbf{4 b}$ - hésitation au niveau du genre des arguments (ex. : *Elle tue le lion; Il *la tue ; Elle *la montre).

$\mathbf{4 c}$ - avec argument/s manquant/s (ex. : $\varnothing+$ tue le lion; Il montre $+\varnothing ;$ Il montre $+\varnothing+$ à la grande sœur ; Il a tué $+\varnothing$ ).

$\underline{\text { niveau } 3}$ - production des erreurs par surgénéralisation

3a - avec arguments présents et justes (ex. : Il *fait montrer le village à Hercule).

$\mathbf{3 b}$ - avec argument/s manquant/s (ex. : Ø+*fait montrer le village à Hercule; Il *fait montrer $+\varnothing ;$ Il lui $*$ fait montrer $+\varnothing)$.

$\underline{\text { niveau } 2}$ - apparition de constructions factitives à la place des causatifs lexicaux (ex. : Il fait mourir le lion; Il a fait tomber le lion, pour tuer).

$\underline{\text { niveau } 1}$ - réponses partielles; il s'agit de réponses portant uniquement sur l'action du causateur (ex. :

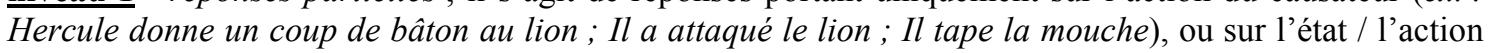
du causataire (ex. : Il est mort, le lion).

$\underline{\text { niveau } 0}$ - absence de réponse cohérente (ex. : Ils regardent toutes les maisons pour voir s'il y a quelque chose.).

Pour coder les comportements des enfants au cours de la tâche de compréhension, nous avons établi une échelle en trois niveaux (de la valeur 2 à la valeur 0). La valeur 2 indique que la construction factitive est disponible en compréhension. Dans ce cas-là, l'enfant exécute l'action signalée par l'enquêtrice ou bien, il la fait exécuter à la figurine de la maman (ex. : L'enfant chatouille la figurine du bébé ou bien l'enfant prend la figurine de la maman et montre à l'enquêtrice comment la maman va chatouiller le bébé, pour le faire rire). La valeur 1 se caractérise par la présence d'un signe de compréhension du prédicat complexe faire + Vinf; autrement dit, l'enfant fournit une explication pertinente de l'action causative sans l'exécuter (ex. : La maman, elle va le taper, pour faire pleurer). La valeur 0 signale l'absence de tout signe de compréhension.

\subsection{Premiers résultats}

Dans cette section, nous présenterons les résultats constatés au stade actuel de notre recherche sur le prédicat complexe faire + Vinf dans le langage des enfants monolingues francophones entre 4 et 6 ans. D'abord, nous rendrons compte du pourcentage de réussite des tâches de production et d'imitation avec les deux cibles faire + Vinf et causatifs lexicaux. Ensuite, nous ferons un bref bilan sur les capacités des enfants à comprendre la construction factitive, ainsi que les verbes tuer et montrer. Enfin, nous comparerons nos résultats actuels avec ceux de l'étude de Sarkar (2002) et sur cette base, nous tenterons de voir si, ou bien, à quel point les étapes de l'acquisition du prédicat complexe faire + Vinf pourraient reproduire celles de sa grammaticalisation.

Les résultats que nous avons obtenus à l'issue des tâches de production et d'imitation avec changement du lexique sont très variés. Au cours de ces deux épreuves, chacun des 40 participants (20 enfants par tranche d'âge, cf. supra) est amené à produire et puis à imiter six constructions factitives (faire rire, faire pleurer, faire tomber, faire danser, faire boire et faire manger) et deux causatifs lexicaux (tuer et montrer). Le tableau 2 ci-dessous résume les catégories de réponses fournies par les enfants des deux tranches d'âge $\left(\mathrm{N}_{1}=4-5\right.$ ans et $\mathrm{N}_{2}=5-6$ ans) avec la cible faire + Vinf: 


\begin{tabular}{|c|c|c|c|c|}
\hline \multirow[t]{2}{*}{ Catégories de réponses } & \multicolumn{2}{|c|}{$\begin{array}{l}\mathrm{N}_{1} \text { (entre } 4 \\
\text { et } 5 \text { ans) }\end{array}$} & \multicolumn{2}{|c|}{$\begin{array}{l}\mathrm{N}_{2} \text { (entre } 5 \\
\text { et } 6 \text { ans) }\end{array}$} \\
\hline & Prod. & Imit. & Prod. & Imit. \\
\hline $\begin{array}{l}5 \mathrm{a}(\text { faire }+ \text { Vinf }+2 \text { arg. justes : Le papa } \\
\text { fait rire la fille })\end{array}$ & 31,7 & 36,7 & 27,5 & 35,8 \\
\hline $\begin{array}{l}5 \mathrm{~b}(\text { faire }+ \text { Vinf }+2 \text { arg. }+ \text { hésitation sur } \\
\text { le genre }: * \text { Elle } * \text { le fait rire })\end{array}$ & 0,8 & 10 & 1,7 & 5 \\
\hline $\begin{array}{l}\text { c }(\text { faire }+\operatorname{Vinf}+2 \text { arg. dont causataire } \\
\text { incorrect : Il *lui fait rire ; Il *la fait } \\
\text { boire du lait })\end{array}$ & 4,2 & 5,8 & 7,5 & 10,8 \\
\hline $\begin{array}{l}5 \mathrm{~d} \text { (faire }+ \text { Vinf }+1 \text { arg. juste }+ \text { arg. } \\
\text { absent : Le papa fait rire }+\varnothing ; \varnothing+\text { lui } \\
\text { fait boire) }\end{array}$ & 4,2 & 7,5 & 5,8 & 5 \\
\hline $\begin{array}{l}\text { 5e (faire }+S N+\text { Vinf: Elle fait *le } \\
\text { pleurer) }\end{array}$ & 0,8 & 0 & 0 & 0 \\
\hline 4a (ellipses : pleurer le bébé) & 0 & 4,2 & 0,8 & 3,4 \\
\hline 4b (ellipses réduites : Tomber) & 0 & 4,2 & 1,7 & 8,3 \\
\hline $\begin{array}{l}\text { 4c (transitivation causative : Il *rit la } \\
\text { fille) }\end{array}$ & 0 & 1,7 & 4,2 & 0 \\
\hline $\begin{array}{l}\text { 3a (donner à manger / boire }+ \text { arg. } \\
\text { présents et justes : Il donne à manger à } \\
\text { la fille) }\end{array}$ & 10,8 & 6,7 & 12,5 & 7,5 \\
\hline $\begin{array}{l}\text { 3b (donner à manger / boire }+ \text { arg. } \\
\text { absent/s : Il donne à manger }+\varnothing ; \varnothing+ \\
\text { lui donne à manger })\end{array}$ & 2,5 & 1,7 & 2,5 & 2,5 \\
\hline $\begin{array}{l}\text { 2a (surgénéralisations : Il *fait casser le } \\
\text { robot) }\end{array}$ & 1,7 & 0,8 & 0 & 0 \\
\hline $\begin{array}{l}2 \mathrm{~b} \text { (surgénéralisations }+ \text { arg. absent } / \mathrm{s}: \\
\text { Il } * \text { fait casser }+\varnothing)\end{array}$ & 0,8 & 0 & 0,8 & 0 \\
\hline \multirow{2}{*}{$\begin{array}{l}1+0 \text { (réponses partielles ou absence de } \\
\text { réponse) }\end{array}$} & 42,5 & 20,7 & 35 & 21,7 \\
\hline & 100 & 100 & 100 & 100 \\
\hline
\end{tabular}

Tableau 2: Pourcentage de réussite des tâches de production et d'imitation avec la cible faire + Vinf

Le tableau 2 ci-dessus montre qu'à ce stade du développement langagier (entre 4 et 6 ans) le prédicat complexe faire + Vinf ne peut pas être considéré comme entièrement maîtrisé.

Quelques tendances principales se font remarquer au cours de la tâche de production. Il s'agit tout d'abord des emplois canoniques de la construction factitive (les arguments causateur et causataire sont présents et justes) ; ceux-ci s'élèvent à $31,7 \%$ chez les enfants entre 4 et 5 ans et à $27,5 \%$ chez les enfants entre 5 et 6 ans (ligne 1 du tableau).

Deuxièmement, les apparitions du prédicat complexe faire + Vinf avec causataire incorrect sont relativement fréquentes; celles -ci sont estimées à 4,2\% chez les plus jeunes locuteurs et à $7,5 \%$ chez les plus âgés (ligne 3 du tableau). Au cours de la dérivation causative, les enfants entre 5 et 6 ans récupèrent souvent le causataire comme datif lorsque le verbe de départ est monovalent (à 1 actant) (ex. : Il rit. - monovalent $\rightarrow$ Il fait rire la fille. $\rightarrow$ Il la fait rire. - bivalent, ce qui sera produit comme Il *lui fait rire), ou bien, ils récupèrent le causataire comme objet lorsque le verbe de départ est bivalent (à 2 actants) (ex. : Il boit du lait. - bivalent $\rightarrow$ Il fait boire du lait à la fille. $\rightarrow$ Il lui fait boire du lait. - trivalent, ce qui sera produit comme Il *la fait boire du lait). 
Dans les productions recueillies, nous avons également relevé des emplois sporadiques de la construction factitive avec un argument juste et un argument absent (ligne 4 du tableau). Le nombre de ces occurrences non conventionnelles s'élève à $4,2 \%$ chez les enfants âgés de 4 à 5 ans et à 5,8\% chez les enfants âgés de 5 à 6 ans.

Tous les participants ont tendance à produire les constructions à deux verbes donner à manger / à boire à la place du prédicat complexe faire + Vinf (ligne 9 du tableau); ces énoncés atteignent 10,8\% chez les enfants entre 4 et 5 ans et $12,5 \%$ chez les enfants entre 5 et 6 ans. Les sujets interviewés manient relativement bien les structures complexes à deux (voire à trois) arguments (ex. : Il donne à manger du

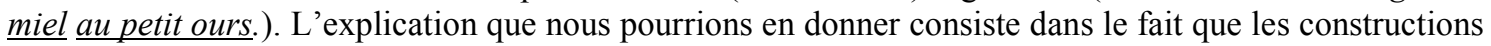
donner à manger / à boire ne sont pas grammaticalisées ; chacun des verbes composants est suivi de son propre argument. En revanche, la fusion des prédicats implique une restructuration, une réanalyse des arguments (le verbe auxiliaire s'attache les clitiques), ce qui retarderait le processus d'acquisition.

Un dernier point sur lequel les énoncés des participants fournis lors de la tâche de production se différencient concerne le nombre des réponses partielles (portant sur l'action du causateur qui fait agir le causataire), ainsi que les cas d'absence de réponse cohérente (qui ne prend en compte aucun des éléments de la situation causative). Ces erreurs sont estimées à $42,5 \%$ chez les plus jeunes locuteurs, tandis que chez les plus âgés elles atteignent $35 \%$ (ligne 13 du tableau). Apparemment, l'émergence de la construction factitive est difficile et, de plus, la situation causative n'est pas toujours conceptualisée. Cette dernière a un caractère particulier : elle ne présente pas un agent exécutant une action ordinaire par luimême (ex. : Jean rit; Jean court.). Elle évoque plutôt l'instigateur, celui qui fait quelque chose pour qu'un autre actant agisse. Il y aurait donc une action intermédiaire (cognitivement présente, mais qui n'est pas exprimée linguistiquement) (ex. : Jean fait rire Marie : 1/action 1 (sous-entendue) = Jean fait une grimace; 2/ action 2 = Marie rit. 3/ Jean fait rire Marie). D'un point de vue cognitif, la présence de cette étape intermédiaire rend la situation causative difficile à conceptualiser, ce qui retarderait l'acquisition de faire + Vinf. Les réflexions de Givón (1991) vont dans le même sens, à savoir que tout ce qui est cognitivement complexe, dans la langue est exprimé par une structure complexe (principe de l'iconicité).

Finalement, nous pourrions dire qu'à ce stade du développement langagier (entre 4 et 6 ans) le prédicat complexe faire + Vinf n'est pas encore entièrement disponible en production. Les emplois canoniques de la construction factitive s'approchent des $30 \%$ chez les représentants des deux classes d'âge. Les enfants commettent toujours des erreurs liées à l'agencement du causataire, ou bien, ils suppriment l'un des arguments de cette structure syntaxique complexe. Lorsqu'il s'agit des verbes manger et boire, les sujets interviewés préfèrent la construction à deux verbes (moins grammaticalisée) donner $\grave{a}+$ Vinf.

Les tendances énumérées ci-dessus réapparaissent au cours de la tâche d'imitation avec la cible faire + Vinf. D'abord, nous constatons une légère augmentation du nombre des exemples conventionnels $(36,7 \%$ chez les enfants entre 4 et 5 ans $v s 35,8 \%$ chez les enfants entre 5 et 6 ans) (ligne 1 du tableau). Ce fait n'est pas surprenant puisque les enfants sont censés produire la construction factitive après avoir entendu le modèle adulte.

Le changement des items lexicaux a un impact considérable sur l'hésitation au niveau du genre des arguments (causateur et causataire). Le degré d'apparition de ces erreurs augmente de 0,8\% (en production) à $10 \%$ (en imitation), chez les enfants entre 4 et 5 ans et de $1,7 \%$ (en production) à $5 \%$ (en imitation), chez les enfants âgés de 5 à 6 ans (ligne 2 du tableau).

Malgré la présence du modèle adulte, nous constatons une hausse du nombre des occurrences avec causataire incorrect ou avec argument absent. Les premières sont estimées à 5,8\% chez les plus jeunes locuteurs vs 10,8\% chez les plus âgés (ligne 3 du tableau). Les secondes atteignent 7,5\% chez les enfants entre 4 et 5 ans, tandis que chez les enfants entre 5 et 6 ans leur nombre baisse à $5 \%$ (ligne 4). Surprenante également est l'apparition des énoncés elliptiques. Chez les participants âgés de 4 à 5 ans, le nombre des ellipses avec un argument présent (le causataire) (ligne 6 du tableau), ainsi que celui de leurs variantes réduites (les deux arguments causateur et causataire sont absents) (ligne 7) augmente de 0 en production à 4,2\% en imitation. Chez les participants âgés de 5 à 6 ans, le nombre des ellipses avec causataire produit (ligne 6) augmente de $0,8 \%$ en production à $3,4 \%$ en imitation; la fréquence des 
énoncés réduits à l'infinitif également accroît (de $1,7 \%$ en production à $8,3 \%$ en imitation (ligne 7 ). Ce phénomène pourrait être dû à des raisons communicatives, c'est-à-dire que l'enfant fait exprès d'éviter la répétition du verbe factitif faire dans ses énoncés, puisque celui-ci est déjà présent dans la question de l'enquêtrice.

En revanche, le nombre des constructions à deux verbes donner à manger / à boire, ainsi que celui des réponses partielles ou nulles diminue en imitation. Les premières atteignent $6,7 \%$ chez les enfants entre 4 et 5 ans et $7,5 \%$ chez les enfants entre 5 et 6 ans (ligne 9 du tableau). Les secondes baissent à 20,7\% chez les plus jeunes locuteurs vs 21,7 \% chez les plus âgés (ligne 13). Apparemment, le modèle adulte fourni par l'enquêtrice oriente les sujets interviewés dans leurs réponses.

Finalement, nous pourrions dire que la présence du modèle adulte fourni par l'enquêtrice au cours de la tâche d'imitation ne garantit pas la parfaite reproduction de la construction factitive faire + Vinf. D'un côté, les erreurs liées au genre des arguments, à l'agencement du causataire, à la présence / absence des actants et du verbe causatif faire persistent. D'un autre côté, le fait de fournir le modèle de formation du prédicat complexe faire + Vinf stimule les enfants à le produire même dans des contextes où ils auraient préféré les constructions à deux verbes donner à manger / à boire ou les réponses partielles.

Il est également intéressant de présenter les performances des enfants entre 4 et 6 ans en production et en imitation avec la cible causatifs lexicaux. Le tableau 3 ci-dessous résume les catégories de réponses obtenues :

\begin{tabular}{|c|c|c|c|c|}
\hline \multirow[t]{2}{*}{ Catégories de réponses } & \multicolumn{2}{|c|}{$\begin{array}{l}\mathrm{N}_{1} \text { (entre } 4 \\
\text { et } 5 \text { ans) }\end{array}$} & \multicolumn{2}{|c|}{$\begin{array}{l}\mathrm{N}_{2} \text { (entre } 5 \\
\text { et } 6 \text { ans) }\end{array}$} \\
\hline & Prod. & Imit. & Prod. & Imit. \\
\hline $\begin{array}{l}\text { 4a (emplois justes: Hercule tue le lion; } \\
\text { Ulysse montre le village à Hercule) }\end{array}$ & 37,5 & 255 & 55 & 42,5 \\
\hline $\begin{array}{l}\text { 4b (hésitation au niveau du genre des } \\
\text { arg. : *Elle *la tue) }\end{array}$ & 0 & 15 & 0 & 12,5 \\
\hline $\begin{array}{l}4 \mathrm{c}(\text { avec arg. absent/s : Il montre }+\varnothing ; \\
\varnothing+\text { tue le lion })\end{array}$ & 15 & 10 & 2,5 & 17,5 \\
\hline $\begin{array}{l}\text { 3a (surgénéralisations: Il *fait montrer } \\
\text { le village à Hercule) }\end{array}$ & 2,5 & 2,5 & 0 & 0 \\
\hline $\begin{array}{l}3 \mathrm{~b} \quad \text { (surgénéralisations avec } \text { arg. } \\
\text { absent/s : Il } * \text { fait montrer }+\varnothing)\end{array}$ & 2,5 & 0 & 2,5 & 0 \\
\hline $\begin{array}{l}2 \text { (constructions factitives: Il fait } \\
\text { mourir le lion) }\end{array}$ & 2,5 & 0 & 10 & 2,5 \\
\hline \multirow[t]{2}{*}{$1+0$ (réponses partielles ou absentes) } & 40 & 17,5 & 30 & 25 \\
\hline & 100 & 100 & 100 & 100 \\
\hline
\end{tabular}

Tableau 3 : Pourcentage de réussite des tâches de production et d'imitation avec la cible causatifs lexicaux

D'après le tableau 3 ci-dessus, en production les performances des enfants entre 5 et 6 ans avec la cible causatifs lexicaux sont meilleures que celles des enfants entre 4 et 5 ans. Les emplois canoniques des verbes tuer et montrer s'élèvent à $55 \%$ chez les locuteurs plus âgés, tandis que chez les plus jeunes ceuxci atteignent 37,5\% (ligne 1). Un autre écart considérable entre les participants des deux tranches d'âge se fait remarquer; il s'agit des occurrences avec argument/s absent/s (15\% chez les enfants entre 4 et 5 ans $v s$ 2,5\% chez les enfants entre 5 et 6 ans) (ligne 3 du tableau). Les erreurs par surgénéralisation dominent également chez les plus jeunes participants (5\% vs 2,5\% chez les plus âgés) (lignes 4 et 5 ). Le nombre des réponses partielles ou nulles reste élevé même avec la cible causatifs lexicaux (40 \% chez les enfants entre 4 et 5 ans $v s 30 \%$ chez les enfants entre 5 et 6 ans) (ligne 7 du tableau). Un dernier point sur lequel les énoncés des participants fournis lors de la tâche de production se différencient concerne 
l'apparition de constructions factitives à la place des verbes tuer et montrer $(2,5 \%$ chez les plus jeunes locuteurs vs $10 \%$ chez les plus âgés) (ligne 6).

De tout ce que nous venons de dire ci-dessus, il en résulte que les enfants entre 5 et 6 ans n'éprouvent pas de difficultés à produire les causatifs lexicaux. En revanche, les enfants entre 4 et 5 ans ont tendance à supprimer certains actants et à commettre des erreurs par surgénéralisation en production. Ils appliquent le schéma faire + , sans tenir compte du fait que dans cette construction, l'espace vide réservé au verbe à l'infinitif ne peut pas être occupé par une variante lexicale du prédicat complexe faire + Vinf (montrer $=$ faire voir).

Les résultats obtenus au cours de la tâche d'imitation avec la cible causatifs lexicaux sont très variés ; Tout d'abord, nous constations une augmentation au niveau des emplois conventionnels des verbes tuer et montrer chez les enfants entre 4 et 5 ans (de $37,5 \%$ en production à $55 \%$ en imitation) (ligne 1 du tableau). En revanche, le nombre de ces exemples canoniques diminue chez les enfants entre 5 et 6 ans (de $55 \%$ en production à $42,5 \%$ en imitation). Un autre fait paradoxal est observable lors de cette tâche expérimentale, à savoir la hausse des occurrences avec argument/s absent/s chez les participants âgés de 5 à 6 ans (de 2,5\% en production à 17,5\% en imitation) (ligne 3 du tableau). Le nombre de ces emplois déviants chez les participants âgés de 4 à 5 ans baisse tout naturellement suite à la présence du modèle adulte (de $15 \%$ en production à $10 \%$ en imitation). Le changement des items lexicaux a un impact considérable sur l'hésitation au niveau du genre des arguments. Ces erreurs sont estimées à $15 \%$ chez les enfants entre 4 et 5 ans et à $12,5 \%$ chez les enfants entre 5 et 6 ans (ligne 2 du tableau). La présence du modèle adulte fourni par l'enquêtrice fait baisser (de $5 \%$ en production à $2,5 \%$ en imitation chez les plus jeunes locuteurs) et disparaître (de 2,5\% en production à 0 en imitation chez les locuteurs plus âgés) les erreurs par surgénéralisation (lignes 4 et 5 du tableau). Il en est de même avec les réponses partielles ou nulles (17,5\% chez les enfants entre 4 et 5 ans $v s 25 \%$ chez les enfants entre 5 et 6 ans) (ligne 7).

De tout ce que nous venons de dire ci-dessus, il s'ensuit que la présence du modèle adulte peut stimuler l'apparition des exemples canoniques des causatifs lexicaux; elle est également capable de faire baisser et même disparaître les erreurs par surgénéralisation. Toutefois, les emplois déviants liés à l'hésitation au niveau du genre des actants et à leur présence / absence persistent.

Notre recherche porte également sur les capacités des enfants entre 4 et 6 ans à comprendre le prédicat complexe faire + Vinf et les causatifs lexicaux tuer et montrer. Le tableau 4 ci-dessous illustre les catégories de comportements constatés chez les participants des deux classes d'âge :

\begin{tabular}{|c|c|c|c|c|}
\hline \multirow{2}{*}{$\begin{array}{l}\text { Catégories de } \\
\text { comportements }\end{array}$} & \multicolumn{2}{|c|}{$\begin{array}{c}\mathrm{N}_{1} \text { (entre } 4 \text { et } 5 \\
\text { ans) }\end{array}$} & \multicolumn{2}{|c|}{$\begin{array}{c}\mathrm{N}_{2} \text { (entre } 5 \text { et } 6 \\
\text { ans) }\end{array}$} \\
\hline & $\begin{array}{c}\text { Faire } \\
+ \\
\text { Vinf }\end{array}$ & $\begin{array}{c}\text { Caus. } \\
\text { lexicaux }\end{array}$ & $\begin{array}{c}\text { Faire } \\
+ \\
\text { Vinf }\end{array}$ & $\begin{array}{c}\text { Caus. } \\
\text { lexicaux }\end{array}$ \\
\hline 2 (compréhension totale) & 50 & 82,5 & 60 & 75 \\
\hline 1 (signe de compréhension) & 12,5 & 0 & 10 & 5 \\
\hline \multirow{2}{*}{$\begin{array}{l}0 \text { (absence de tout signe de } \\
\text { compréhension) }\end{array}$} & 37,5 & 17,5 & 30 & 20 \\
\hline & 100 & 100 & 100 & 100 \\
\hline
\end{tabular}

Tableau 4 : Pourcentage de réussite de la tâche de compréhension avec les cibles faire + Vinf et causatifs lexicaux

D'après le tableau 4 ci-dessus, ce sont les enfants entre 5 et 6 ans qui fournissent les meilleurs résultats en compréhension avec la cible faire + Vinf. (60\% vs $50 \%$ chez les plus jeunes) (ligne 1 du tableau). Les participants âgés de 4 à 5 ans donnent souvent des signes de compréhension sans exécuter l'action causative (ex. : Elle l'a tapé, la maman, en réponse à La maman fait pleurer le bébé. A toi maintenant, fais comme la maman!) (12,5\% vs $10 \%$ chez les plus âgés) (ligne 2). L'absence de tout signe de 
compréhension est également plus fréquente chez les enfants entre 4 et 5 ans $(37,5 \%$ vs $30 \%$ chez les enfants entre 5 et 6 ans) (ligne 3 du tableau).

Les participants âgés de 4 à 5 ans se montrent meilleurs en compréhension avec la cible causatifs lexicaux (82,5\% vs $75 \%$ chez les plus âgés) (ligne 1 du tableau). En revanche, les explications des actions causatives, ainsi que l'absence de tout signe de compréhension dominent chez les enfants entre 5 et 6 (lignes 2 et 3 ).

Finalement, nous pourrions dire qu'à ce stade du développement langagier, la construction factitive et les causatifs lexicaux sont disponibles en compréhension. Le prédicat complexe faire + Vinf est cognitivement présent, puisque les enfants âgés de 4 à 6 ans se montrent capables de le reconnaître dans l'environnement langagier (l'input).

Les démarches méthodologiques, que nous avons adoptées pour mener notre expérimentation, ainsi que nos premiers résultats vont au-delà de ceux rapportés par Sarkar (2002). Elle explore la capacité des jeunes locuteurs (entre $1 ; 9$ et $3 ; 10$ ans) à comprendre et à produire la construction factitive (spontanément, ou bien, après avoir entendu le modèle adulte). L'analyse quantitative qu'elle applique lui permet d'esquisser les trois principales étapes dans l'acquisition du PC faire + Vinf (p. 191) :

a) le verbe factitif faire est souvent omis (ex. : Je danse le petit chat.). L'auteur précise qu'entre la période de suppression massive de faire (avant 2 ans) et celle de son apparition consécutive, il y aurait une étape intermédiaire au cours de laquelle certains enfants sont plutôt disposés à le supprimer, tandis que d'autres sont plutôt disposés à le produire (mostly omitting or mostly supplying) (p. 180).

b) clitiques mal placés, alors faire + Vinf n'est pas encore considéré comme PC, mais comme une périphrase (ex.: *Je fais les sauter.). Sarkar précise que ce type d'erreurs est rare dans son corpus. De plus, le mauvais placement des clitiques est toujours accompagné par de nombreux exemples d'emplois conventionnels.

c) construction stabilisée (ex. : Puis, je vais le faire sauter sur le bébé.).

Notre corpus contient très peu d'occurrences confirmant les deux premières étapes - omission relative de faire et interprétation bi-clausale (à deux prédicats distincts) de la construction faire + Vinf. Ce fait serait dû à l'âge plus avancé de nos sujets interviewés (entre 4 et 6 ans). En revanche, les données que nous avons recueillies et traitées à l'heure actuelle de notre recherche vont à l'encontre de l'affirmation de Sarkar (2002), selon laquelle l'émergence du prédicat complexe faire $+\operatorname{Vinf}$ se fait entre 2 et 4 ans. La maitrise de la construction factitive en production atteint $31,7 \%$ chez les enfants âgés de 4 à 5 ans vs 27,5 $\%$ chez les enfants entre 5 et 6 ans; la présence du modèle adulte en imitation fait augmenter le nombre des emplois canoniques du prédicat complexe faire + Vinf à 36,7\% chez les plus jeunes locuteurs $v s$ 35,8 $\%$ chez les plus âgés. Par ailleurs, cette structure syntaxique est disponible en compréhension $(62,5 \%$ chez les participants entre 4 et 5 ans vs $70 \%$ chez les participants entre 5 et 6 ans).

Les enfants entre 4 et 5 ans fournissent les meilleurs résultats (excepté en compréhension), quoique l'écart entre les productions des représentants des deux tranches d'âge n'est pas important. Ce phénomène qui paraît paradoxal pourrait être expliqué par la tendance à la créativité, à la diversification des moyens d'expression chez les enfants entre 5 et 6 ans. En revanche, les plus jeunes locuteurs, qui maîtrisent moins les outils de la langue chercheraient plutôt à reproduire le modèle adulte.

\section{Conclusion}

Les résultats actuels de notre étude sur l'acquisition du prédicat complexe faire + Vinf nous permettent de tirer quelques conclusions intéressantes.

Premièrement, nous ne disposons d'aucun exemple confirmant l'hypothèse que les enfants interprètent précocement la construction factitive faire + Vinf comme une seule unité prédicative. Ce fait n'a rien de surprenant vu l'âge de nos sujets interviewés (de 4 à 6 ans). Pour les mêmes raisons, à notre avis, les 
données que nous avons recueillies et traitées à l'heure actuelle ne prouvent pas non plus l'existence d'une période où les enfants interprètent faire + Vinf comme une construction à deux verbes distincts. Les seules apparitions de structures complexes moins grammaticalisées dans le langage enfantin concernent donner à manger / à boire, mais ces constructions sont également à deux prédicats chez les adultes.

Deuxièmement, les résultats dont nous disposons nous laissent supposer qu'à ce stade du développement langagier (entre 4 et 6 ans) la construction factitive faire $+\operatorname{Vinf}$ en français n'est pas encore acquise. Elle est sans aucun doute cognitivement présente et, par conséquent, facile à reconnaître dans l'environnement langagier (l'input). Toutefois, en production le prédicat complexe faire $+\operatorname{Vinf}$ est en voie de stabilisation; cette étape du développement langagier est marquée par quelques tendances générales, à savoir : 1/ emploi canonique de la construction factitive ; 2 / hésitation au niveau du genre des arguments (causateur et causataire), ainsi qu'au niveau des fonctions syntaxiques du causataire (objet ou datif) ; 3/ suppression d'un argument ; 4/ apparition de la construction à deux verbes donner à + Vinf à la place du prédicat complexe faire + Vinf.

Finalement, les résultats actuels de la recherche que nous avons menée ne révèlent aucune correspondance entre les étapes de grammaticalisation et celles d'acquisition du prédicat complexe faire + Vinf. Le processus diachronique se caractérise par le passage d'une construction à deux prédicats (en latin) à une seule unité prédicative (en français contemporain). Dans certains cas la grammaticalisation aboutit à l'apparition de variantes lexicales des constructions factitives (montrer - faire voir). Pour ce qui est du processus acquisitionnel, se fiant aux résultats fournis par Sarkar (2002), au départ, les enfants interprètent faire + Vinf comme une seule unité prédicative, d'où l'apparition des exemples de transitivation causative (emploi transitif, causatif ${ }^{+}$d'un verbe intransitif). L'évolution de faire + Vinf dans le langage enfantin se poursuit par une courte période de fluctuation entre construction à deux prédicats et prédicat complexe pour aboutir à une structure syntaxique stabilisée de fort degré de grammaticalisation. Il serait intéressant de mener des études plus approfondies sur une éventuelle correspondance entre grammaticalisation et acquisition du prédicat complexe faire + Vinf auprès des enfants d'âges variés.

\section{Références bibliographiques :}

Abeillé, A. \& Godard, D. (2003). Les prédicats complexes. Les langues romanes. Problèmes de la phrase simple, Sous la dir. de D. Godard, Paris : CNRS (éd)., 1 - 62.

Alsina, A. (1996). The role of argument structure in grammar. Evidence from romance. Stanford, CA: CSLI Publications.

Ammon, M.S. \& Slobin, D.I. (1978). A cross-linguistic study of the processing of causative sentences. Papers and Reports on Child Language Development, 15, 114 - 128.

Bowerman, M. (1974). Learning the structure of causative verbs : A study in the relationship of cognitive, semantic and syntactic development. Papers and Reports on Child Language Development, 8, $142-178$.

Chamberlain, J.T. (1986). Latin Antecedents of French Causative faire. New York / Bern / Frankfurt : Lang.

Cicéron. (I ${ }^{\mathrm{er}}$ s. av. J.-C.). Epistulae ad Atticum. T. 2, Livres VII-XI. Paris : Garnier (1938).

Combettes, B. et al. (2003). Introduction : grammaticalisation et changement linguistique. Verbum, XXV, 2003, 3, $225-240$.

Comrie, B. (1976). The syntax of causative constructions: cross-language similarities and divergences. In Shibatani, M. (éd.), Syntax and semantics, Volume 6: The grammar of causative constructions, New York: Academic Press, $261-312$.

Creissels, D. (2006). Syntaxe générale: une introduction typologique. T. 1. Paris: Lavoisier.

Dixon, R.M.W. (2000). A typology of causatives : form, syntax, meaning. In Dixon, R.M.W. \& Aikhenvald, A. (éd.), Changing valency. Case studies in transitivity, Cambridge: Cambridge University Press, $30-83$. 
Gaatone, D. (1976). Les pronoms conjoints dans la construction factitive. Revue de Linguistique romane, $n^{\circ} 159-$ $160,165-182$.

Givón, T. (1991). Markedness in Grammar: Distributional, Communicative and Cognitive Correlates of Syntactic Structure. Studies in Language, vol. 15, $n^{\circ} 2,335-370$.

Gougenheim, G. (1929). Etude sur les périphrases verbales de la langue française. Paris : Les Belles lettres.

Heine, B. (1993). Auxiliaries. Oxford: Oxford University Press.

Hopper, P. (1987). Emergent grammar. Berkeley Linguistics Society, 13, 139 - 157.

Hopper, P.J. \& Traugott, E.C. (1993). Grammaticalization. Cambridge: Cambridge University Press.

Kurylowicz, J. (1965). The evolution of grammatical categories. Esquisses linguistiques, 1975, 38 - 54.

Labelle, M. (1984). Acquisition des constructions causatives par des apprenants de français langue seconde. Unpublished master's thesis, Université d'Ottawa.

La Chanson de Roland. Paris : GF-Flammarion.

Lamiroy, B. (1999). Auxiliaires, langues romanes et grammaticalisation. Langages, 135, 33 - 45.

Levin, B. \& Rappaport Hovav, M. (1995). Unaccusativity. At the Syntax-Lexical Semantics Interface. Cambridge (Mass.); London: MIT Press.

Lucrèce Carus. (Ier s. av. J.-C.). De rerum natura. T. 1, Livres I-III. Paris : Les Belles Lettres (1968).

Marchello-Nizia, C. (2001). Grammaticalisation et évolution des systèmes grammaticaux. Langages, 130, 33 - 41.

McDaniel, D. \& McKee, C.\& Smith Cairns, H. (1998). Methods for assessing children's syntax. Cambridge (Mass.); London: MIT Press.

Meillet, A. (1912 / 1982). L'évolution des formes grammaticales. Linguistique historique et linguistique générale. Paris : Champion, 1948, $130-148$.

Nilsson-Ehle, H. (1948). Observations sur la soudure syntaxique du groupe «Faire + Infinitif ». Studia linguistica, Année II, 93 - 118.

Perlmutter, D. (1978). Impersonal Passives and the Unaccusative Hypothesis. Proceedings of the Berkeley Linguistic Society 4, $157-189$.

Peyraube, A. (2002). L'évolution des structures grammaticales. Langages, 146, 46 - 58.

Plaute. (IIIe - IIe s. av. J.-C.). Comédies (Epidicus). T. 3. Paris : Les Belles Lettres (1935).

Sarkar, M. (2002). Saute ça / "Jump this!": The acquisition of the faire faire causative by first and second language learners of French. Annual Review of Language Acquisition, 2, 157 - 201.

Shibatani, M. (1976). The grammar of causative constructions : a conspectus. In Shibatani, M. (éd.), Syntax and semantics, Volume 6: The grammar of causative constructions. New York: Academic Press, $1-40$.

Simone, R. \& Cerbasi, D. (2001). Types and diachronic evolution of Romance causative constructions. Romanische Forschungen, 113, $441-473$.

Tomasello, M. (2003). Constructing a language: a usage-based theory of language acquisition. Cambridge, Massachusetts, and London, England: Harvard University Press.

Virgile. (I ${ }^{\text {er }}$ s. av. J.-C.). Enéide. T. 1, Livres I-IV. Paris : Les Belles Lettres (1992). 
Psycholinguistique, acquisition

DOI $10.1051 / \mathrm{cmlf0} 222$

1 C'est également le cas en français contemporain (ex. Il fait rire la fille. - faire + verbe intransitif; La maman fait manger du gâteau au bébé. - faire + verbe transitif).

2 Dans l'exemple (5) le verbe causatif faire est suivi de l'infinitif d'un verbe transitif.

3 Dans l'exemple (6) le verbe causatif faire est suivi de l'infinitif d'un verbe intransitif.

4 Les exemples de (8) à (10) sont enmpruntés à Gougenheim (1929 : 357 - 358).

5 Les exemples de (11) à (14) sont empruntés à Gougenheim (1929: 331, 358).

6 Par exemple, Gaatone (1976: 166 - 169) propose quelques tests qui rapprochent la construction factitive des formes verbales, composées d'un auxiliaire (avoir/être) et d'un participe passé ; ceux-ci fournissent une preuve convaincante en faveur du PC. Ici, nous en citerons quelques-uns : 1/ l'insertion des clitiques de l'objet entre le sujet et faire +Vinf (ex. : Elle fait manger le bébé $\rightarrow$ Elle le fait manger, *Elle fait le manger), 2/ l'impossibilité de supprimer l'infinitif (ex. : Elle a fait manger l'enfant. *Elle l'a fait $+\varnothing), 3 /$ la forme négative (ex. : Elle ne fait pas manger le bébé. *Elle ne fait manger pas le bébé.), etc.

7 Dans une correspondance avec A. Gide, Nilsson-Ehle lui demande «si, en écrivant des phrases du genre [ «Ce besoin de noblesse qui, passé vingt-six ans, fait encore Nietzsche préférer Schiller à Gœthe »], il a eu l'intention de ranimer la vieille manière de s'exprimer, ou si [...] ces phrases lui sont venues au bout de la plume, naturellement (et avec le seul souci de la précision de la pensée). » (Nilsson-Ehle, $1948: 101$ ). A ce questionnement A. Gide répond de la manière suivante : "N'en doutez pas : c'est tout naturellement que je me suis exprimé ainsi-sans même trop me rendre compte $[\ldots] »($ idem $)$.

8 Les exemples de (15) à (16) sont empruntés à Gougenheim (1929:358).

9 Nous précisons que les procédures de recueil et de traitement des données sont en cours. Le nombre total des participants prévu pour notre recherche s'élève à 60 enfants monolingues francophones. Le présent article est basé sur la partie déjà analysée du corpus recueilli.

10 Sur le plan syntaxique, les verbes inergatifs agentifs dont rire, pleurer, jouer se caractérisent par une valence stable ; ceux-ci désignent une action qui part du sujet (plan sémantique). Pour ces raisons, il est impossible de les utiliser sans un verbe causatif (ex. : The teacher *played the children.).

11 Nous n'avons trouvé aucun emploi transitif (causatif + ) de ce verbe en français, tandis que l'anglais se montre plus souple à son égard autorisant la suppression de l'auxiliaire causatif make sous certaines conditions (ex. : Dance me to the end of love.).

12 Sur le plan syntaxique, les verbes inaccusatifs de changement d'état ou de position dont tomber se caractérisent par une valence instable ; ceux-ci expriment une action qui vise ou atteint le sujet (plan sémantique). Pour ces raisons, il serait possible de les utiliser sans un verbe causatif (ex. : Aujourd'hui, il faut bouger les jeunes.). Des emplois transitifs (causatif + ) du verbe tomber en français sont attestés au Moyen âge et au XVI ${ }^{\mathrm{e}}$ siècle (Gougenheim, 1929 : 324). 